\title{
LOW COST-LOW SPEED OSCILLOSCOPE USING GRAPHICAL LCD AND ATmega128 MICROCONTROLLER
}

\author{
Sanchit Sachdeva ${ }^{1}$, Ramandeep Singh ${ }^{2}$ \\ ${ }^{I} P G$ Student M.Tech (ES), EECE Department, ITM University, Gurgaon, Haryana, India \\ ${ }^{2}$ Assistant Professor, EECE Department, ITM University, Gurgaon, Haryana, India
}

\begin{abstract}
In this paper, we have discussed the development of existing digital oscilloscope which is used for research and development purpose, instead of using cathode ray oscilloscope we have realized a circuit using ATmegal28 IC [1]. To convert costly device affordable and which consume less space and less power leads to save time and money. In this way we have try to overcome the cost and space occupied by oscilloscope.
\end{abstract}

Keywords: Oscilloscope, Graphical LCD, AVR Microcontroller, LM358 Comparator, cost effective, compact size, embedded system, research and development.

\section{INTRODUCTION}

An oscilloscope, formerly known as oscillograph, and informally it is known as a CRO (cathode ray oscilloscope).It is an electronic instrument which is used to test the observation of constantly varying signal voltages [2]. Circuit operating voltage is $12 \mathrm{~V}$ DC. These observations are shown in the form of tow dimensional graph i.e. one or more electric potential difference is shown using the y-axis or vertical axis where as function of time is plotted against the x-axis or horizontal axis. The classical oscilloscope using cathode ray is difficult to frame at home because of size, high voltage presence and mechanical fragility [4]. Alternative solution to above problem is modern PC having the advantage of post processing the signal and its recording capabilities but this is also an expensive and dangerous if PC is not isolated from its chassis. Another solution to this is manufacturing of digital oscilloscope using GLCD screen.

\section{BLOCK DIAGRAM}

Given block diagram is used to explain the basic functioning of oscilloscope using ATmega128 and GLCD screen. We have a battery source which will provide us a $12 \mathrm{~V}$. This voltage is divided into two different voltage values i.e. $8.2 \mathrm{~V}$ and $5 \mathrm{~V}$. Input signal and $8.2 \mathrm{~V}$ is given to LM358 IC which is used as a comparator $.5 \mathrm{~V}$ is used to powered up microcontroller ATmega128 and GLCD screen [8]. We have interface the switches for increasing and decreasing the time. Similarly for beam up and beam down. Switch $1 \& 4$ are used for decreasing and increasing the time or frequency. Similarly switch $2 \& 5$ are used to move beam up and down. Switch 3 is used to freeze the beam. Output from LM358 is given to micro-controller and we have used in built ADC to convert the analog signal to digital signal using sampling and quantization [2] which is then showed in the GLCD screen [7].

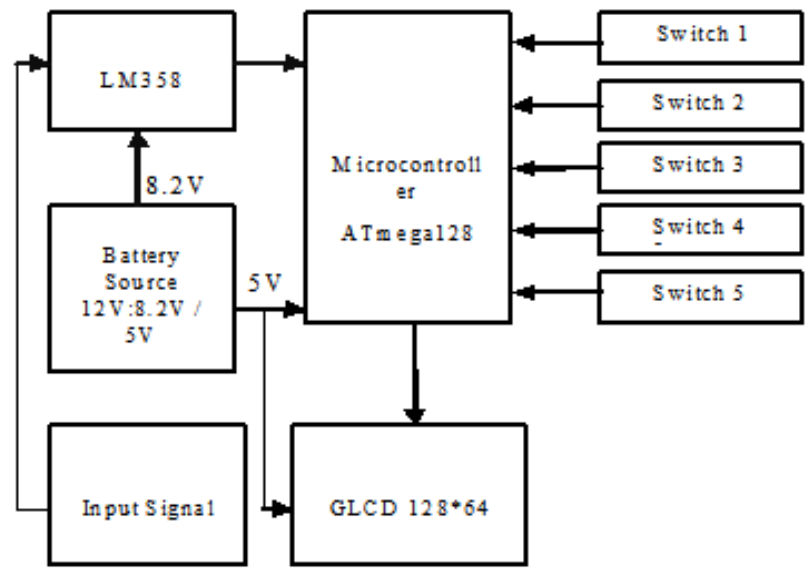

Fig-1: Block Diagram of Low Speed AVR Oscilloscope

\section{FLOW CHART}

The system will work as follow:

Analog Signal from the device is fed to the LM358 Comparator. LM358 work as a differential amplifier and compares the differential voltage with the reference voltage. We are using $12 \mathrm{~V}$ DC battery as a Source of Energy and $12 \mathrm{~V}$ is divided into $5 \mathrm{~V}$ and $8.2 \mathrm{~V}$. LM358 is provided with $8.2 \mathrm{~V}$ of source energy and it work as a reference voltage.LM358 then sample and compare the analog signal with $8.2 \mathrm{~V}$ of source energy [6]. After that analog signal is fed to the ADC which is inbuilt in the ATMega128 microcontroller [1][9].ADC converts the analog signal to digital signal by sampling and quantization[2].Then Changes has been done as per the requirement of the user with the use of switches. Then signal is displayed on Grapical LCD[3][9]. 


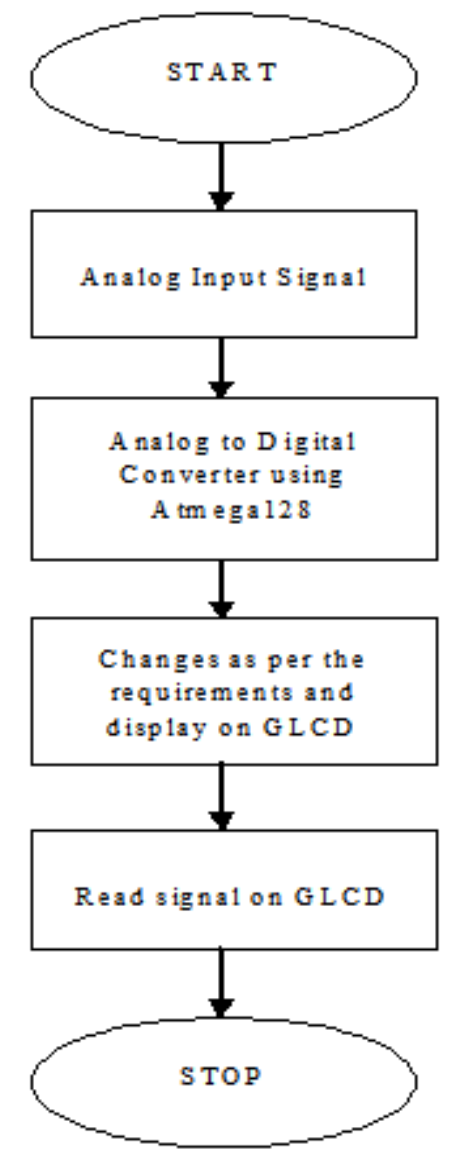

Fig-2: Flow Chart of the designed Oscilloscope

\section{ALGORITHM}

Analog signal is fed to LM358 which work as differential amplifier and compares the differential voltage with the reference voltage i.e. 8.2V.Differential Amplifier uses the negative feedback to improve the output performance of the system. After that analog to digital converter in AVR samples and calculate the middle value of the signal wave form. Samples are calculated from one full period. AVR microcontroller [1] samples the signal continuously and each sample is compared with the previous one. If the current sample value is higher than the previous sample then waveform result as rising waveform and AVR will go to step 4. If the current sample value is lower than the previous value then AVR will go to step 2. Again calculate the value of next sample and compared it with the middle value. If the value of next sample is higher then the middle value then its a rising waveform and AVR will calculate the waveform upto the selected pixels. AVR thus Prints those samples on GLCD[8][9]. AVR starts again from step 1.

\section{GRAPHICAL LCD}

GLCD is used to display customized characters and symbols but in character lcd we can display only characters and numeric numbers. We are using GLCD $128 * 64$ in which we have 128 columns and 64 rows and 128*64 means 1024 pixels [7]. GLCD has max. of 20 pins.

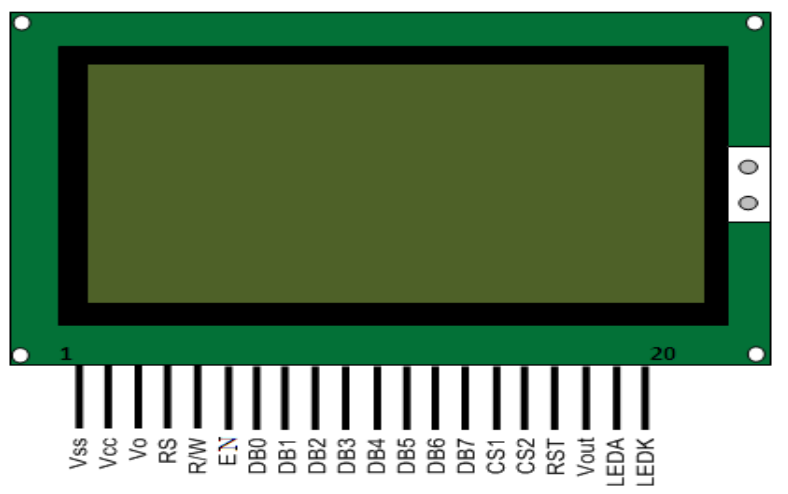

Fig -3: GLCD with 20 pins

Data port is of 8 bits and it works on $+5 \mathrm{~V}$. GLCD is used where we want character and graphical representation to display. RS,RW and Enable are the control pins of the GLCD Each half has 8 pages and it is controlled by a separate controller. In each page we have 8 rows and 64 columns. For selecting the halves we have two pins named as chip select [7]. Graphical LCD is mainly used in many applications like mobile phones, video games, lifts as a display. GLCD is divided into two equal halves [8].

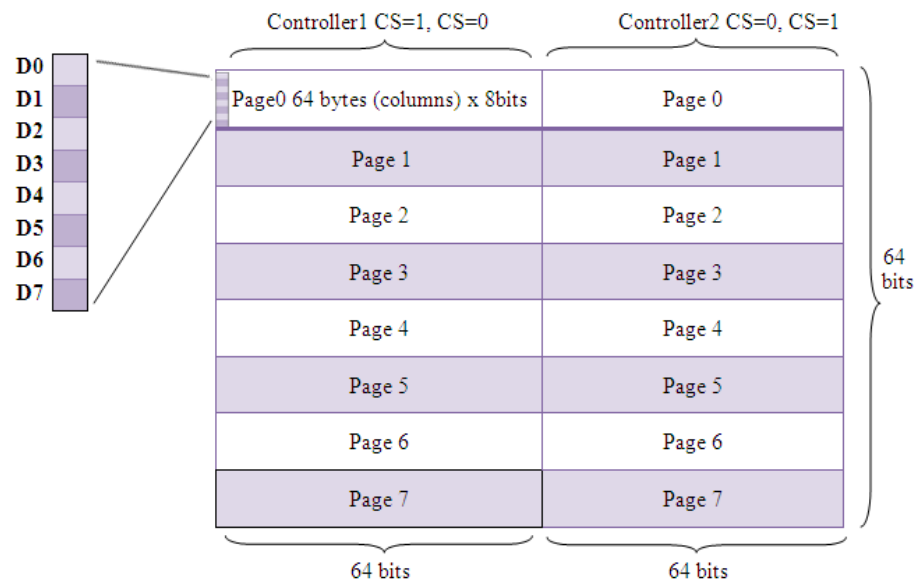

Fig-4: Page format of GLCD

Above figure shows the division of two halves. Each half consist of 8 pages and each half is selected by using the chip select pin 15 and 16 of GLCD [8]. If high is given to pin 15 and low is given to pin 16 then first half is selected and vice versa.

\section{GLCD SCREEN}

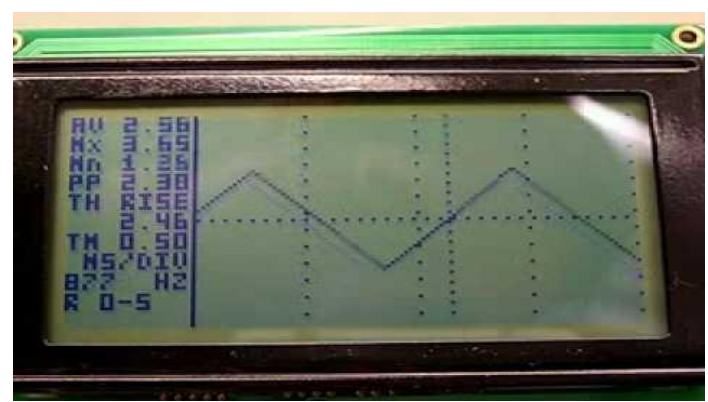

Fig-5: Triangular Waveform displayed on GLCD 
Above figure shows the triangular waveform displayed on glcd. Triangular wave is not an pure sine wave and. it is known as non sinusoidal waveform. It contains odd harmonics because its symmetry is odd. In this each successive harmonics starts from its opposite phase.

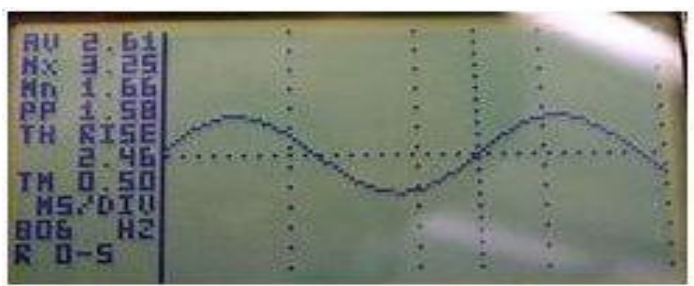

Fig-6: Sine Waveform displayed on GLCD

Above figure shows the sine wave displayed on glcd. It is also known as sinusoidal because it has a repetitive oscillations. Amplitude of sine wave is max. distance it reaches from zero and frequency is defined as the cycle it completes per second. Sine wave from is described as a sine function studied in plane trigonometry.

\section{ADVANTAGES}

Major advantage of using GLCD is that everything is represented using pixels,so detailed information can be calculated or observed. GLCD [7] have a brighter and bigger display as comparison to charcter LCD so it can display waveforms and other symbols which can't be display using character LCD. Equivalent time sampling and consecutive samples lead to higher resolution down to controller. This device takes less space than the ideal oscilloscope, also it consumes less power. Also slow variations like the temperature variation across a day can be recorded [11].Using of low impedance power supply help us to reduce the spikes.

\section{DISADVANTAGES}

Proper isolation is required from the Power-supply so that we should obtain good resolution or signals. Electromagnetic noise from the circuit should be reduced [5],because of the small bandwidth we can't expect the signal above the bandwidth. As there is no proper calibration is done so we get the square wave with little round edges because high frequency components are lost. Switching devices, LCD and micro-controller uses the same digital ground so there will be switching supply noise [6] [7]. Therefore, ADC should be isolated from this noise. All though system shares the same VDD so there will be some noise.

\section{APPLICATIONS}

We can calculate the frequency of an oscillating signal and also we can measure voltage in range from 0 to $+5 \mathrm{~V}$.By using probe with 1:10 division you can measure almost 10 times higher voltages [4]. Imp. application of device is differential measurement. To measure the bandwidth first calculate the max. gain.

\section{CONCLUSIONS}

Thus, this system is capable of displaying different waveforms by using ATmega128 and graphical LCD. It has remove the problem of moving the ideal oscilloscope from one place to another because of its size and it is affordable too. Due to small size it is easy to handle and operate, therefore this system can be used in research purposes and development. Therefore, this system can be used as portable device.

\section{ACKNOWLEDGEMENTS}

I am very thankful to EECE Dept.ITM University for helping me in understanding the different system of research and conceptual problems.

\section{REFERENCES}

[1]. Kaifeng's ATMEGA32 principles and development [M].Beijing: Electronic Industry Press, 2005.P156-178

[2]. Banff, Alberta Canada "Equivalent Sampling Oscilloscope with External Delay Embedded System," hpcc - ices , pp.195-201, 2011 IEEE International Conference on High Performance Computing and Communications, 2011 September 02-September 04.

[3]. Yang Liu, Rue Rodriguez and Johan Liu, "Design for Manufacturability and Reliability of Embedded Display Smart Card",High Density Microsystems Design and Packaging and Component Failure Analysis, 2005. HDP '05. Proceeding of the 7th IEEE CPMT Conference on , 27 June30 June 2005.

[4]. K. L. Shepard, Y. Zheng, "On-Chip Oscilloscopes for Noninvasive Time-Domain Measurement of Waveforms," iccd, pp.0221, 2001 IEEE International Conference on Computer Design (ICCD'01), 2001 September 23September 26.

[5]. Duane, J., Asplundh, J., Liu, J., "Microsystems manufacturing, assembly and packaging technology in Display-Card/spl trade/ applications", High Density Microsystems Design and Packaging and Component Failure Analysis, 2004. HDP '04. Proceeding of the Sixth IEEE CPMT Conference on , 30 June-3 July 2004.

[6]. Zhenjiang Yang. A / D, D / A converter interface technology and practical line [M]. Xi'an: Xidian UniversityPress, 2002: P198-218.

[7]. Hua Fang, Hua Jiang, "The Research on the Algorithm of Lattice LCD Drawing Controlled by PIC," dcabes, pp.226-229, 2011 10th International Symposium on Distributed Computing and Applications to Business, Engineering and Science, 2011

[8]. R. Ladanyi, P. Miklosi, "Functionality Test of End-ofLife TFT LCD Panels," isee, pp.351-355, Proceedings of the 2006 IEEE International Symposium on Electronics and the Environment, 2006.

[9]. Jidan Al-Eryani, Dietmar Nitzsche, Sebastian vSattler, "A System-Level Modelv for an Analog-to-Digital Converter," ims3tw, pp.100-105, 2011 IEEE 17th International Mixed-Signals, Sensors and Systems Test Workshop, 2011. 


\section{BIOGRAPHIES}

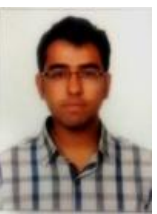

Sanchit Sachdeva is currently Post Graduate student in Masters of Technology, Embedded Systems from ITM University, Gurgaon. He completed his B.Tech in Electronics and Communication Engineering from Akal College of Engineering \& Technology, Eternal University, Himachal Pradesh in 2012. His area of interest is Embedded System Design and Real Time Systems.

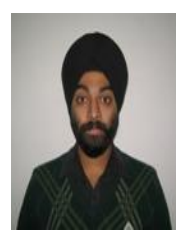

Ramandeep Singh is an Assistant Professor in EECE Department of ITM University, Gurgaon. He is pursuing Ph.D in embedded systems from ITM University. In 2009 he has completed M.E. in embedded systems. He is a B. Tech. graduate from GGSIPU, Delhi. His core research areas are low power embedded systems, robotics, FPGA based embedded systems and SCADA. Prior to joining ITM University he has worked with NXP Semiconductors, Bangalore. He has various publications in international journals and conferences. 